\title{
Dielectrophoretic investigations of sub-micrometre latex spheres
}

\section{N G Green and H Morgan}

Bioelectronics Research Centre, Department of Electronics and

Electrical Engineering, Rankine Building, Oakfield Avenue, University of Glasgow, Glasgow G12 8LT, UK

Received 21 April 1997

\begin{abstract}
A non-uniform AC electric field induces a motion in polarizable particles, called dielectrophoresis. The force responsible for this motion is governed by the dielectric properties both of the suspending medium and of the particles, as well as the geometry of the field. The dielectrophoretic properties of sub-micrometre latex spheres have been studied using micro-fabricated electrode structures. The electric field geometry for electrodes used in the measurements has been solved using numerical analysis. Measurements of the dielectrophoretic properties of the spheres have been made over a range of medium conductivities and applied field frequencies and strengths. Comparisons between the observed behaviour and that expected from theory are presented.
\end{abstract}

\section{Introduction}

Dielectrophoresis (DEP) is the motion of polarizable particles in non-uniform alternating electric fields and arises from the interaction of the field and the induced dipole [1]. It has become an important field of biotechnological research and has been reviewed in detail [2-5].

The DEP force can be derived from the time-dependent force equation

$$
\boldsymbol{F}(t)=(\boldsymbol{m}(t) \cdot \nabla) \boldsymbol{E}(t)
$$

where $\boldsymbol{E}(t)$ is the time-dependent electric field and $\boldsymbol{m}(t)$ is the dipole moment which, for a spherical particle, can be expressed in a frequency-dependent form as

$$
\boldsymbol{m}(\omega)=4 \pi \varepsilon_{m} r^{3} f_{C M} \boldsymbol{E}
$$

where $\omega$ is the angular field frequency, $r$ is the particle's radius and $f_{C M}$ is the Clausius-Mossotti factor given by

$$
f_{C M}=\frac{\varepsilon_{p}^{*}-\varepsilon_{m}^{*}}{\varepsilon_{p}^{*}+2 \varepsilon_{m}^{*}}
$$

where $\varepsilon_{p}^{*}$ and $\varepsilon_{m}^{*}$ are the complex permittivities of the particle and the medium respectively. For a real dielectric, the complex permittivity is

$$
\varepsilon^{*}=\varepsilon-\mathrm{j} \frac{\sigma}{\omega}
$$

where $\mathrm{j}=\sqrt{-1}, \varepsilon$ is the permittivity and $\sigma$ is the conductivity of the dielectric.
The DEP force is given by the real part of (1) and, in time-averaged form, is

$$
\boldsymbol{F}(\omega)=2 \pi \varepsilon_{m} r^{3} \operatorname{Re}\left(f_{C M}\right) \nabla\left|E_{r m s}\right|^{2} .
$$

From equation (5), it can be seen that the DEP force depends on the term $\nabla\left|E_{r m s}\right|^{2}$, (a factor related to the geometry of the electric field) and on the real part of $f_{C M}$, the in-phase component of the particle's effective polarizability. The real part of $f_{C M}$ is bounded by the limits $1<\operatorname{Re}\left(f_{C M}\right)<-\frac{1}{2}$ and varies with the frequency of the applied field and the complex permittivity of the medium. Positive DEP occurs when $\operatorname{Re}\left(f_{C M}\right)>0$, the force is towards increasing field strength and the particles collect at the electrode edges where the highest field strengths are found. The converse of this is negative DEP, in which the force is in the direction of decreasing field strength, the particles being repelled from the electrode edges. The frequency dependence of $f_{C M}$ makes DEP a powerful means of manipulating particles in solution.

Because dielectrophoresis is a non-invasive, nondestructive technique, it has potential uses in a wide range of biotechnological applications and, as recent publications have shown, the separation and concentration of microorganisms and mammalian cells is possible [6-14]. Microand nano-fabrication methods used in the semiconductor industry allow small electrodes to be manufactured [15]. The high electric field strengths generated by such electrodes have allowed DEP manipulation both of viruses and of sub-micrometre latex spheres [16-21].

In this paper, the results of dielectrophoretic investigations of the behaviour of sub-micrometre latex spheres are 


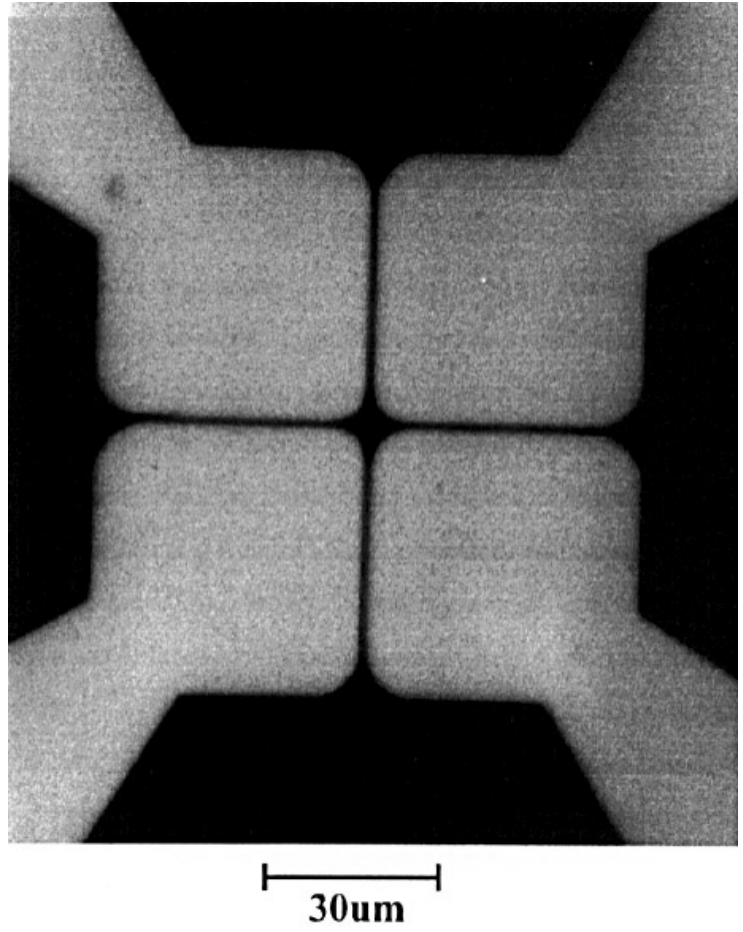

Figure 1. A photograph of a polynomial electrode array used for dielectrophoretic manipulation of latex spheres taken with a light microscope $(\times 40)$. The distance across the centre between opposing electrode pairs is $6 \mu \mathrm{m}$ and the parallel gap between adjacent electrodes is $2 \mu \mathrm{m}$.

presented, together with analysis of the data in terms of simple theoretical models.

\section{Materials and methods}

\subsection{Electrodes}

The 'polynomial' design, shown in figure 1, has been described in the literature [22] and its properties studied experimentally and theoretically [23]. This design produces not only steep gradients at the high-field points but also steep gradients directed towards a low-field 'trap' in the centre. Particles can therefore be collected either by positive or by negative DEP.

The electrode-manufacturing procedures and the experimental equipment and methodology have been explained in detail previously $[18,19]$. The electrodes were manufactured on glass microscope slides and consisted of $10 \mathrm{~nm}$ titanium, $10 \mathrm{~nm}$ palladium and $100 \mathrm{~nm}$ gold. They were made using standard photolithographic and electron-beam lithographic techniques. For DEP studies of 557 and $282 \mathrm{~nm}$ diameter spheres, electrodes were fabricated with a $6 \mu \mathrm{m}$ gap between opposing electrode tips and a $2 \mu \mathrm{m}$ gap between adjacent electrodes.

\subsection{The electric-field analysis}

Electric field plots, showing the three-dimensional variation of the field strength around the electrodes, were calculated using finite-element field-analysis software (Maxwell 3D,
Ansoft Corp, Pittsburgh, USA). The general operating principle of the software is as follows: the solution space is divided into a tetrahedral mesh and the potential is calculated at each vertex and the mid-point of every side from Poisson's equation. The potential is approximated by a low-order polynomial so that the electric field across each tetrahedron can be calculated, with the accuracy of the solution controlled by the number of elements in the mesh. The software performs error analysis on the field in each tetrahedron and then refines the mesh where the error is greatest, increasing the number of elements until the desired solution accuracy is reached.

The symmetry of the polynomial design used (shown in figure 1) meant that only one electrode of the four needed to be modelled in the calculation. An electrostatic approximation was assumed and the surface of the electrode was modelled as a Dirichlet boundary (electric field normal) with a potential of $5 \mathrm{~V}$. The electrode was modelled sitting on the interface of two $20 \mu \mathrm{m}$ cubes, the upper cube representing water and the lower cube consisting of glass (the substrate). The boundary conditions of the sides of the cubes were set to mirror the electrode to produce a full solution. Typically, the number of elements in the final solution was approximately 50000 .

\subsection{Latex spheres}

Latex spheres 282 and $557 \mathrm{~nm}$ in diameter were obtained from Molecular Probes (Oregon, USA). The spheres were carboxylate-modified with a net negative surface charge and had been pre-loaded with a yellow-green fluorescent dye which allowed single-particle observation by fluorescence microscopy. For DEP experiments, the spheres were centrifuged and washed three times in either 1 or $10 \mathrm{mM}$ potassium phosphate buffer at $\mathrm{pH}$ 7.2. They were then resuspended to a final concentration of $0.02 \%$ by volume for the experiments.

A scanning electron micrograph of the 282 and of the $557 \mathrm{~nm}$ diameter spheres is shown in figure 2. The distribution in particle size was estimated from images such as these and was found to be in accord with the manufacturer's specification of $282 \mathrm{~nm} \pm 2.6 \%$ and $557 \mathrm{~nm} \pm 2 \%$. The electrophoretic mobility of a suspension of spheres was measured using a Coulter particle analyser and a distribution in mobilities was observed for both particle sizes. Using the Helmoltz-Smoluchowski equation [24] and the Gouy-Chapman and Grahame theory of the double layer [25], this spread of mobilities was translated into a spread in the surface charge density [18]. The surface charge density of the $557 \mathrm{~nm}$ spheres was given by the manufacturer as $-1.85 \mathrm{C} \mathrm{m}^{-2}$ and measured to be $-0.041 \pm 0.006 \mathrm{C} \mathrm{m}^{-2} \dagger$ and that for the $282 \mathrm{~nm}$ spheres was given as $-0.0483 \mathrm{C} \mathrm{m}^{-2}$ and measured to be $-0.031 \pm 0.006 \mathrm{C} \mathrm{m}^{-2}$; see table 1 .

$\dagger$ The relatively large discrepancy between our measured values and the manufacturer's is due to the fact that they do not directly measure the surface charge on the beads; the data are supplied to them and errors can occur. 


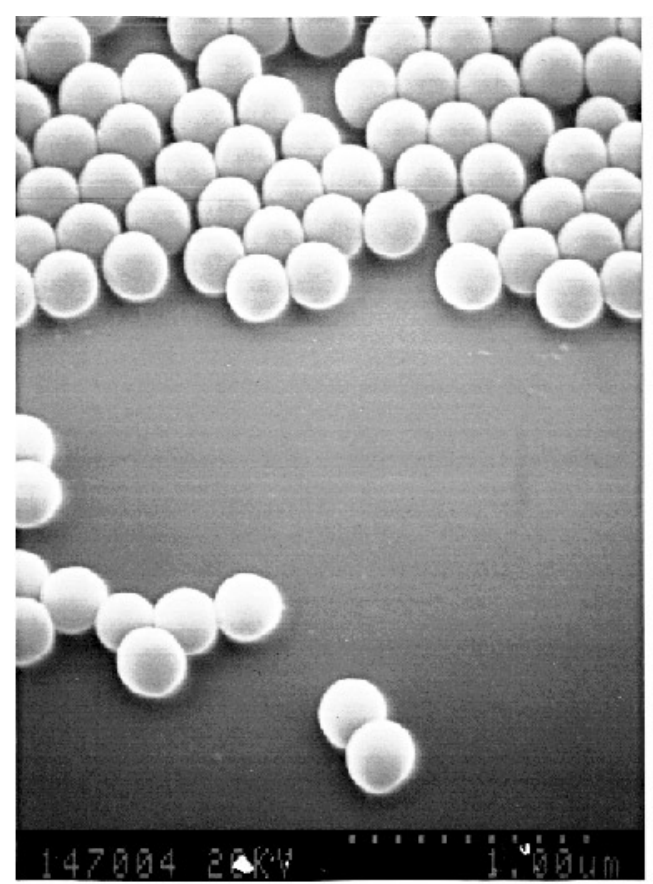

(a)

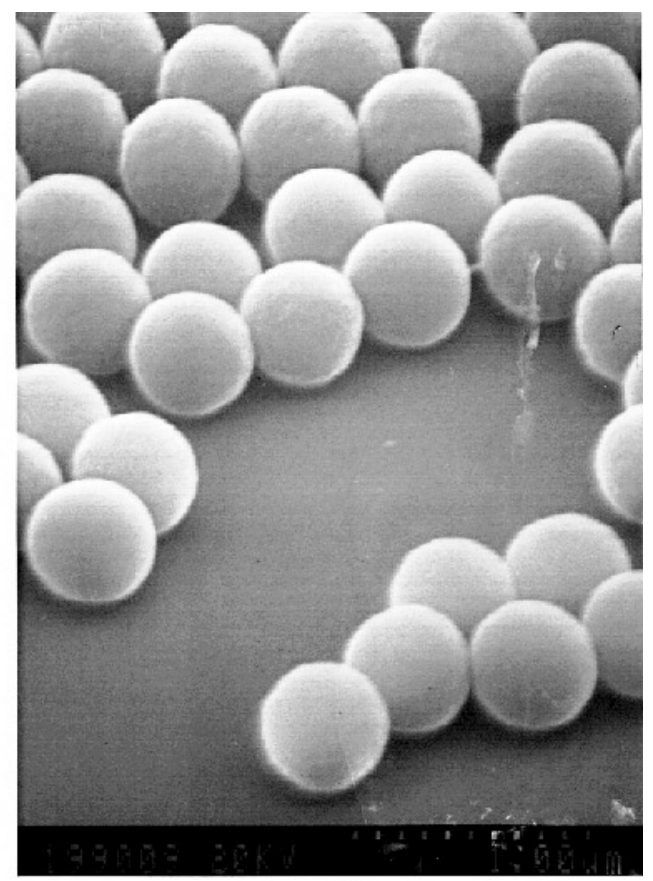

(b)

Figure 2. Scanning electron microscope images of the two sizes of latex spheres used for DEP experiments. The $282 \mathrm{~nm}$ diameter spheres are shown in (a) and the $557 \mathrm{~nm}$ ones in (b).

Table 1.

\begin{tabular}{lccc}
\hline Sphere size $(\mathrm{nm})$ & 282 & \multicolumn{2}{c}{557} \\
Surface charge density $\rho_{b}$ (measured) $\left(\mathrm{C} \mathrm{m}^{-2}\right)$ & $-0.031 \pm 0.006$ & $-0.041 \pm 0.006$ \\
Zeta potential (calculated, see text) $(\mathrm{mV})$ & $46.3 \pm 5.7$ & $-54.9 \pm 4.1$ \\
Buffer concentration $(\mathrm{mM})$ & 10 & 1 & 10 \\
Conductivity $\sigma_{b}\left(\mathrm{~S} \mathrm{~m}^{-1}\right)$ & 0.17 & 0.0185 & 0.17 \\
Debye length $\kappa^{-1}(\mathrm{~nm})$ & 3.05 & 9.65 & 3.05 \\
Measured crossover frequency $(\mathrm{kHz})$ & $70-200$ & $150-300$ & $20-250$ \\
Relaxation frequency (calculated) & & & \\
Schwarz, equation $(8)(\mathrm{kHz})$ & 31 & 8 & 8 \\
Lyklema et al, equation $(9)(\mathrm{kHz})$ & $146 \pm 13$ & $118 \pm 8$ & $43 \pm 2.7$ \\
\hline
\end{tabular}

\section{Results}

\subsection{The electric-field analysis}

The electric-field distribution in the polynomial electrodes was calculated for potential differences of $0.1,1$ and $10 \mathrm{~V}$ between adjacent electrodes. Typical results for a potential of $10 \mathrm{~V}$ are shown in figure 3. Figures 3(a)-(d) are plots of the electric field magnitude, at heights of $0,0.1,1$ and $5 \mu \mathrm{m}$ above the electrode surface.

Figure 4 is a pseudo-three-dimensional plot of $\boldsymbol{E}^{2}$, with the data plotted in the $x-y, x-z$ and $y-z$ planes. Figure 4 has been drawn with the origin at the centre of the four electrodes (see figure 1). From equation (5), it can be seen that the DEP force is proportional to the gradient of the field squared for a given particle; figure 4 represents the potential energy for negative DEP in the centre of the polynomial electrode array.

\subsection{Dielectrophoresis}

Spheres were suspended in a range of molarities of potassium phosphate buffer at $\mathrm{pH}$ 7.2. The buffer conductivities were measured using a Hewlett-Packard bridge at $10 \mathrm{kHz}$ to $10 \mathrm{MHz}$ using a platinum-black cell with a cell constant of $1 \mathrm{~cm}^{-1}$. The $1 \mathrm{mM}$ buffer had a conductivity of $0.018 \mathrm{~S} \mathrm{~m}^{-1}$ and the $10 \mathrm{mM}$ one, $0.17 \mathrm{~S} \mathrm{~m}^{-1}$. The frequency of the applied field was varied in the range $1 \mathrm{kHz}$ to $20 \mathrm{MHz}$ and the potential from $0.1 \mathrm{~V}$ peak-peak to $10 \mathrm{~V}$ peak-peak. The experiments were observed by fluorescence microscopy and recorded on S-VHS video.

It was observed that the dielectrophoretic properties of the spheres varied with the applied frequency and the medium conductivity. Figure 5(a) is a fluorescence photograph showing $282 \mathrm{~nm}$ spheres responding under positive dielectrophoresis and being attracted to the highfield regions at the electrode edges. This photograph was taken with an applied voltage of $4 \mathrm{~V}$ peak-peak at $100 \mathrm{kHz}$ 


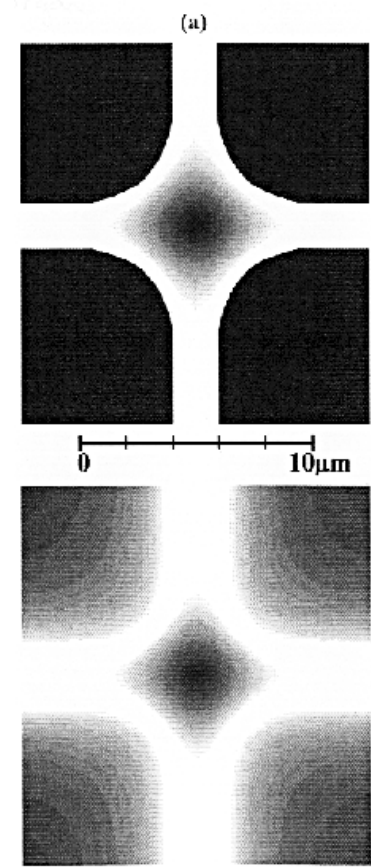

(b)
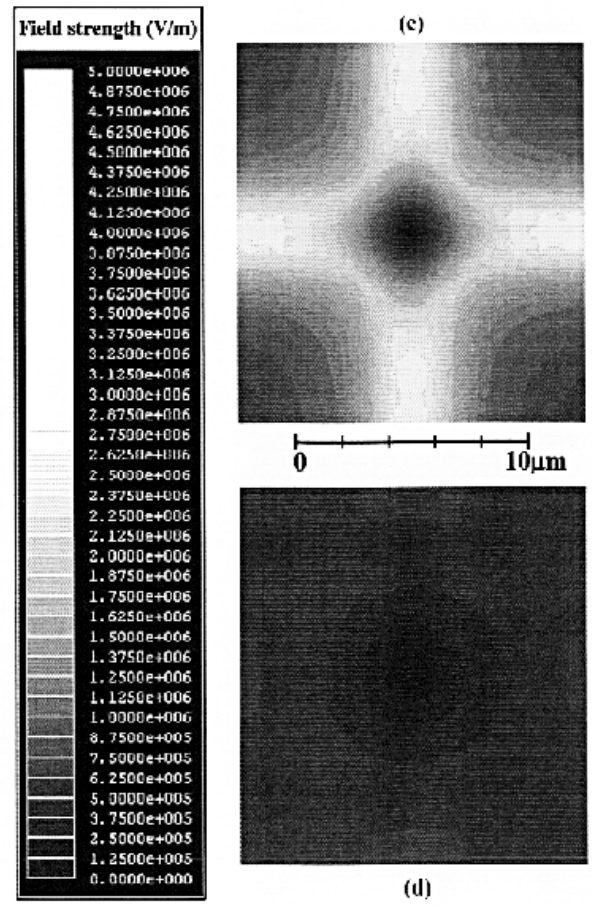

(d)

Figure 3. A plot of the electric-field distribution for the polynomial electrodes shown in figure 1, for an electrode potential of $10 \mathrm{~V}$ peak-peak. The plot shows the field magnitude in planar slices parallel to the electrodes at (a) $0 \mu \mathrm{m}$, (b) $0.1 \mu \mathrm{m}$, (c) $1 \mu \mathrm{m}$ and (d) $5 \mu \mathrm{m}$ above the top of the electrode surface.

and the medium conductivity was $0.17 \mathrm{~S} \mathrm{~m}^{-1}$. Figure 5(b) shows the $282 \mathrm{~nm}$ spheres trapped in the centre of the electrode array by negative DEP in the same buffer. The applied voltage was unchanged at $4 \mathrm{~V}$ peak-peak but the applied frequency was increased to $1 \mathrm{MHz}$. It is clear from these two photographs that the dielectrophoretic response of the spheres varies with applied frequency at a constant medium conductivity. Similar results were obtained in the $0.018 \mathrm{~S} \mathrm{~m}^{-1}$ conductivity buffer and for the $557 \mathrm{~nm}$ spheres.

Figure 6 shows the frequency ranges over which positive DEP, or negative DEP and no movement were observed for the particles. Figures 6(a) and (b) summarize results for the $282 \mathrm{~nm}$ spheres in 1 and $10 \mathrm{mM}$ potassium phosphate buffer respectively and figures $6(\mathrm{c})$ and (d) show summary results for the $557 \mathrm{~nm}$ spheres in the same buffer concentrations. It can be seen from figure 6 that in general, positive DEP occurs from the lowest frequencies measured $(10 \mathrm{kHz})$ up to a frequency in the range $100 \mathrm{kHz}$ to $5 \mathrm{MHz}$ depending on the experimental conditions. Negative DEP was observed for frequencies above a crossover point (up to approximately $20 \mathrm{MHz}$ ) which depended on the medium conductivity and the particle size. For the $282 \mathrm{~nm}$ spheres in $0.018 \mathrm{~S} \mathrm{~m}^{-1}$ buffer the crossover point was well defined and found to cover the range 4-6 MHz. However, for the higher conductivity buffer and also for the $557 \mathrm{~nm}$ spheres, a spread in the crossover frequencies was measured. At any given frequency within this region, individual spheres were seen moving either to the lowor to the high-field regions (positive and negative DEP respectively). For the $557 \mathrm{~nm}$ spheres in $0.17 \mathrm{~S} \mathrm{~m}^{-1}$ buffer this effect occurred over almost two decades of frequency, (figure (d)).

\section{Discussion}

The field plot in figure 3 shows that there was a deep potential minimum in the centre of the polynomial electrodes where the electric field varied from zero at the centre to in excess of $5 \times 10^{6} \mathrm{~V} \mathrm{~m}^{-1}$ at the electrode edges. Figure 4 shows that the square of the electric field, $\boldsymbol{E}^{2}$, increased rapidly near the edges of the electrodes and consequently the gradient of $\boldsymbol{E}^{2}$ acted to trap particles in the potential energy minimum at the centre of the electrode. These results are in qualitative agreement with previous calculations for the dielectrophoretic potential in a plane above the polynomial electrodes [26]. Figure 4 also shows that, as the particles levitate above the surface, the maximum trapping force decreases rapidly.

The experimental results (such as figure 5) are in accordance with the electric field simulations. Under conditions of positive DEP, the spheres collect at the electrode edges, pearl chaining across the $2 \mu \mathrm{m}$ gap (figure 5(a)). Negative-DEP trapping occurs as expected in the centre of the electrodes (figure 5(b)).

The crossover frequency from positive to negative DEP occurs when the real part of equation (3) equals zero. For the $282 \mathrm{~nm}$ spheres in a buffer of conductivity $0.018 \mathrm{~S} \mathrm{~m}^{-1}$ this occurred at a frequency of $5 \pm 1 \mathrm{MHz}$. Taking the permittivity of latex to be $\varepsilon_{p}=2.5$, the conductance of the particle can be estimated from equation (3) to be $0.0185 \mathrm{~S} \mathrm{~m}^{-1}$. The conductivity of a particle can be written as the sum of a bulk conductance $\left(\sigma_{b}\right)$ and a surface 


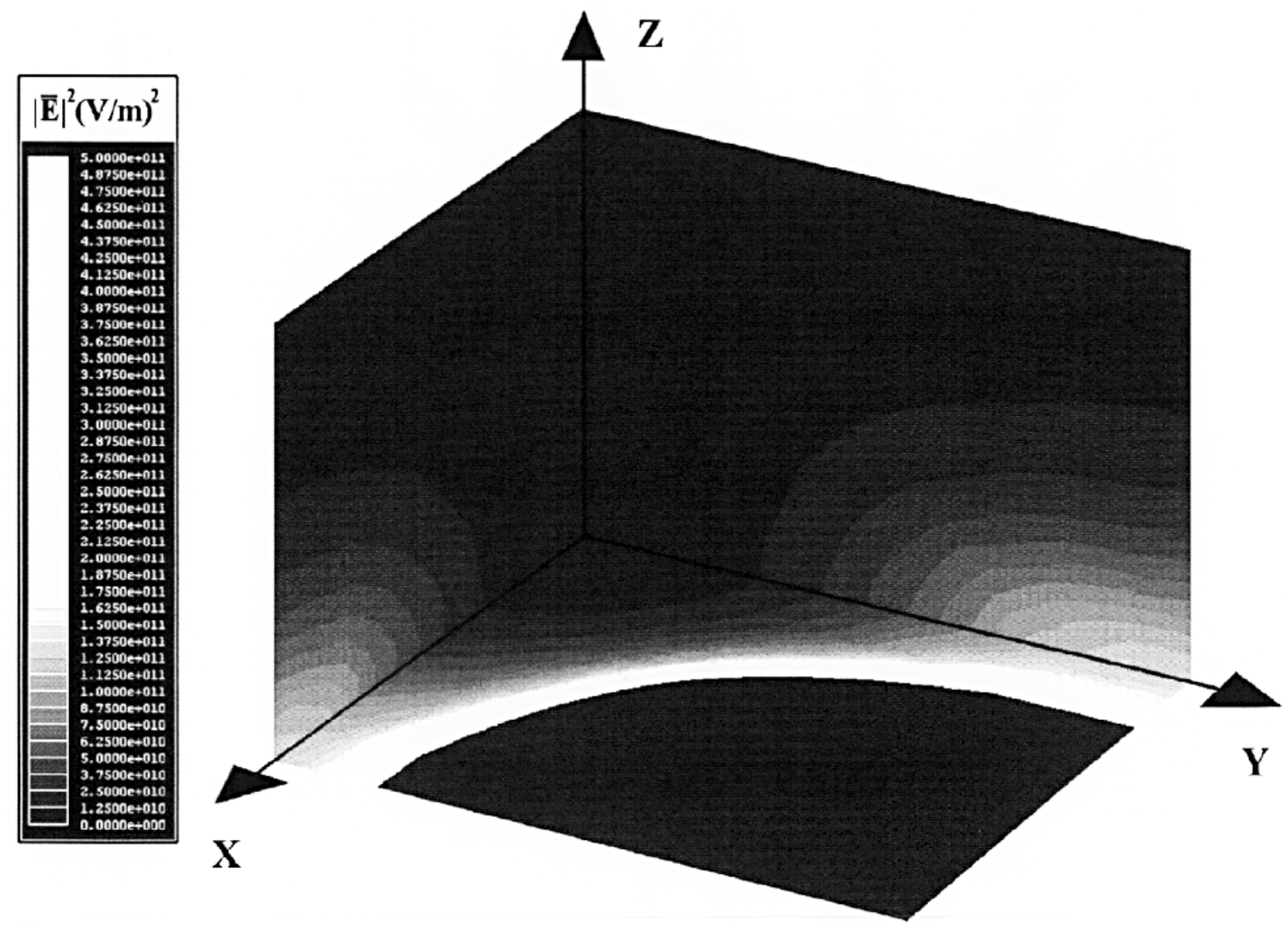

Figure 4. A representation of the DEP force which gives rise to the trap in the centre of the electrodes. Taking the origin at the centre of the electrodes (in 3D), $\boldsymbol{E}^{2}$ was plotted in the $x-y, x-z$ and $y-z$ planes. The DEP force is proportional to the gradient of this plot and in the planes shown there is no normal component to the force vector. The plot therefore represents a complete solution for the force in the planes and shows the variation of the force with the height above the electrodes as well as with the distance from the centre.

conductance $\left(\kappa_{s}\right)[27,28]$. Therefore, the total conductivity $\sigma_{p}$ can be written

$$
\sigma_{p}=\sigma_{b}+\frac{2 \kappa_{s}}{a}
$$

where $a$ is the radius of the particle. For the latex spheres $\sigma_{b} \cong 0$, so that, from our data for the $282 \mathrm{~nm}$ spheres, the surface conductance can be estimated to be $1.3 \mathrm{nS}$, a value which is in general agreement with literature data on latex spheres of larger sizes [29].

For a single homogeneous solid dielectric sphere immersed in a conducting medium the dielectrophoretic properties are generally attributed to a single dielectric relaxation due to Maxwell-Wagner interfacial polarization, known as the $\beta$-relaxation [30]. The frequency of this dispersion is given by

$$
\tau_{\beta}=\frac{1}{2 \pi f_{\beta}}=\frac{\varepsilon_{p}+2 \varepsilon_{m}}{\sigma_{p}+2 \sigma_{m}} .
$$

Owing to the relatively small forces imparted on submicrometre spheres our observations of the positive and negative DEP regimes were obtained with approximately equal forces either side of the crossover point. Generally, the crossover frequency for a relaxation due to interfacial polarization occurs within half a decade or so of the frequency calculated from equation (7). Therefore, the measured crossover frequencies which are close to those predicted by equation (7) can be attributed to the MaxwellWagner relaxation frequency.

For the $282 \mathrm{~nm}$ spheres in the low-conductivity buffer, the Maxwell-Wagner relaxation frequency was calculated to be $f_{\beta}=6.5 \mathrm{MHz}$, which is very close to the observed crossover frequency of $5 \mathrm{MHz}$. For the solution of higher conductivity $\left(0.17 \mathrm{~S} \mathrm{~m}^{-1}\right) f_{\beta}=42.3 \mathrm{MHz}$ (calculated), compared with an observed crossover frequency in the range 0.07 to $0.2 \mathrm{MHz}$. Assuming a surface conductance of $1 \mathrm{nS}$, then calculations for the Maxwell-Wagner relaxation frequency for the $557 \mathrm{~nm}$ spheres give similar frequencies, whereas measured crossover frequencies are at least two decades lower, see table 1.

Dielectric measurements for suspensions of charged latex spheres [31,32] indicate the existence of two dispersions. The high-frequency dispersion is generally assigned to the Maxwell-Wagner interfacial polarization mechanism and the second dispersion, which occurs at a lower frequency, has been interpreted as a relaxation of the electrical double layer associated with the surface charge of the sphere. From the early work of Schwarz [28], the 


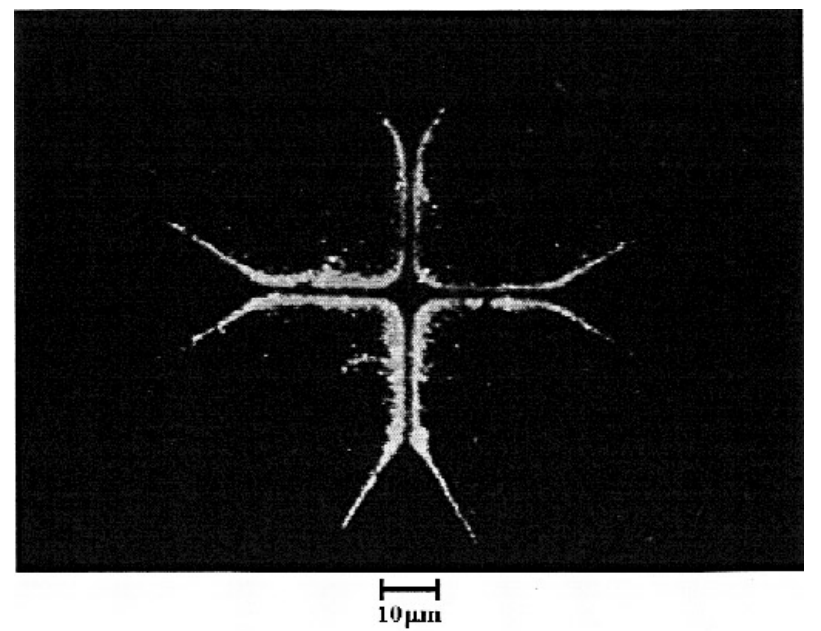

(a)

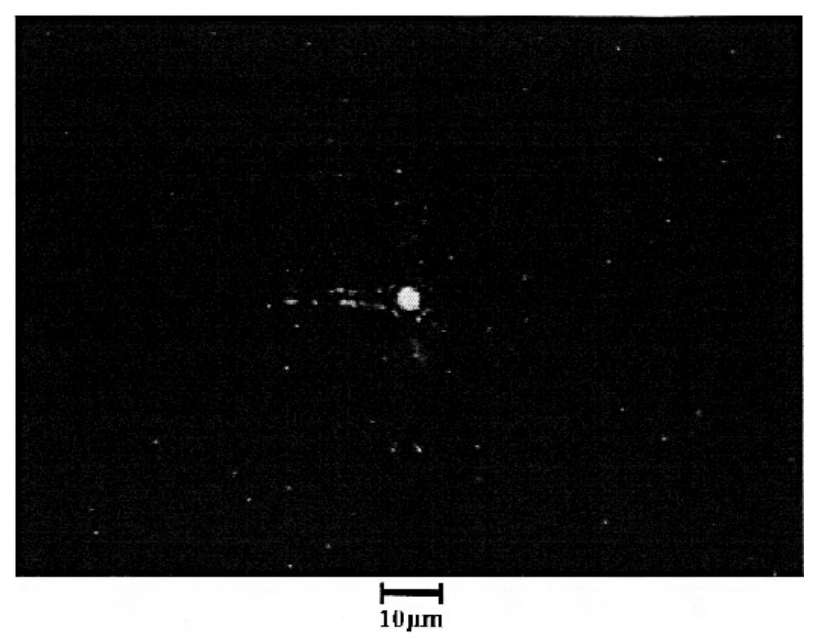

(b)

Figure 5. Digitized video images of fluorescently loaded $282 \mathrm{~nm}$ diameter latex spheres observed with a fluorescence microscope: (a) spheres trapped by positive DEP at $100 \mathrm{kHz}$ and an applied voltage of $4 \mathrm{~V}$ peak-peak and (b) spheres undergoing negative DEP and remaining trapped in a potential energy minimum at the centre of electrode array; the frequency in this case was $1 \mathrm{MHz}$ and the applied voltage $4 \mathrm{~V}$ peak-peak. The suspending buffer was $10 \mathrm{mM}$ potassium phosphate, of conductivity $0.17 \mathrm{~S} \mathrm{~m}^{-1}$.

relaxation time for this dispersion is given as

$$
\tau_{\alpha}=\frac{a^{2}}{2 D}
$$

where $a$ is the particle radius and $D$ is the diffusion coefficient of the ions in the electrical double layer. Lyklema, Duhkin and Shilov introduced a comprehensive theory [33-35] to account for the low-frequency relaxation of the double layer, involving either polarization of the diffuse component or polarization of the bound layer of charge. In the latter case the equation for the relaxation time is similar to that of Schwarz but modified to include a factor, $M$, which takes into account the contribution from the polarization of the bound layer of charge. The characteristic relaxation frequency for this dispersion is then

$$
\tau_{\alpha}=\frac{a^{2}}{2 D_{b}} \frac{1}{M}
$$

with $D_{b}$ the diffusion coefficient of the bound counterions and $M$ defined as

$$
M=1+\frac{F \rho_{b}}{R T C_{d}} .
$$

The differential capacitance of the double layer is given by

$$
C_{d}=\varepsilon_{0} \varepsilon_{r} \kappa \cosh \left(\frac{F \Phi_{d}}{2 R T}\right) .
$$

$\kappa$ is the reciprocal Debye length, $\rho_{b}$ the bound surface charge density, $\Phi_{d}$ the zeta potential and other symbols have their usual meaning.

On the basis of our measurements for the $1 \mathrm{mM}$ buffer, $M \approx 14.8$ (282 $\mathrm{nm}$ spheres) and for the $10 \mathrm{mM}$ buffer, $M \approx 4.7,(282 \mathrm{~nm}$ spheres) and $M \approx 5.3$ (557 nm spheres). It is likely that the bound ion diffusion constant and the permittivity of the medium will have values less than the bulk values. As an approximation, using the bulk values for medium permittivity together with the value of the diffusion constant of free potassium ions $\left(D=1.94 \times 10^{-9} \mathrm{~m}^{2} \mathrm{~s}^{-1}\right)$, the frequencies predicted by this equation were calculated and are given in table 1 . These calculations point to the fact that the measured crossover frequencies for the $282 \mathrm{~nm}$ spheres in $10 \mathrm{mM}$ potassium phosphate buffer and the $557 \mathrm{~nm}$ spheres in both buffer concentrations must be attributable to the dispersion of the electrical double layer rather than to interfacial polarization mechanisms.

The large overlap in crossover frequencies in figure 6 can be attributed to a number of factors such as the distribution in surface charge on the particles and a spread in particle diameter. However, the spread in sphere diameters is typically $\pm 2 \%$ around the mean, which gives an uncertainty of $3-4 \%$ in the frequencies. This may add to, but cannot account for, the observed distribution in crossover frequencies. The origin of the distribution is likely to be a wide spread in surface charge density on individual spheres. The $\pm 10 \%$ spread in calculated relaxation frequencies shown in table 1 was derived from the width at half height of a near-Gaussian distribution in measured surface charge density. Such a variation has been exploited to perform separation of a mixture of nanoparticles with a range of surface charge into two populations [18].

The movement of particles in the electrodes is affected by a number of forces apart from DEP. The high electric fields used in the experiments would be expected to cause localized heating of the sample [20]. This effect is governed by the electrohydrodynamic equations which describe not only the thermally driven but also the electrically driven convective liquid streaming [36]. Convection due to heating forms cylindrical vortices over electrode edges and several other effects are observed which occur at frequencies which change with particle size. At certain frequencies, electroconvection completely dominates the particle behaviour. A typical example of particle flow under 
(a) $282 \mathrm{~nm}$ spheres in $1 \mathrm{mM}$ potassium phosphate

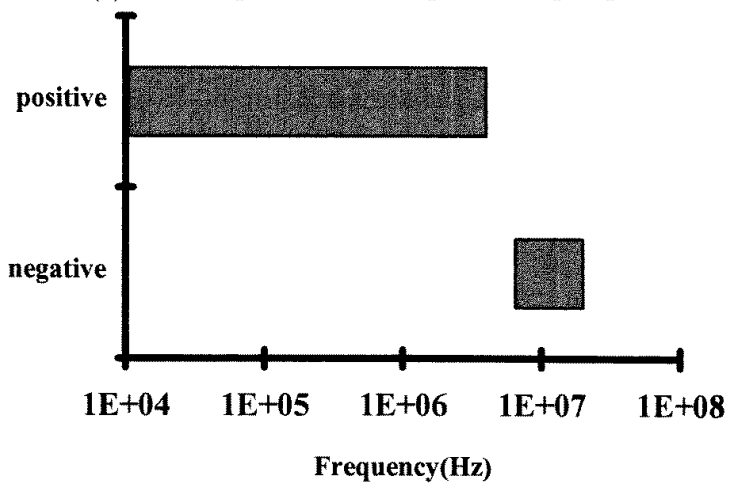

(c) $557 \mathrm{~nm}$ spheres in $1 \mathrm{mM}$ potassium phosphate

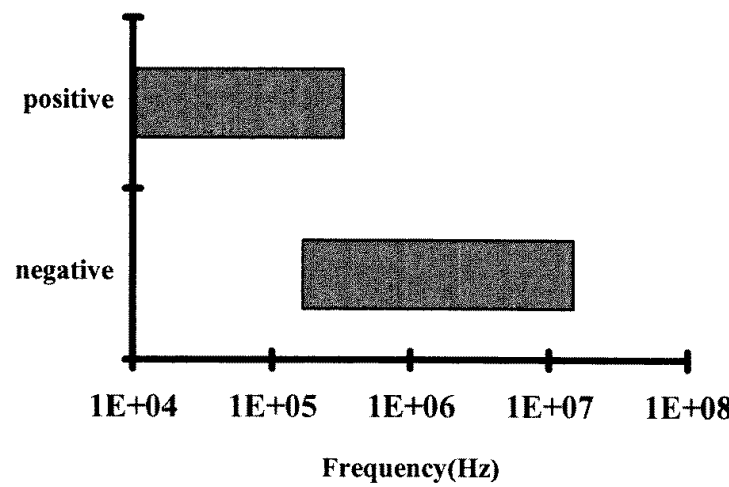

(b) $282 \mathrm{~nm}$ spheres in $10 \mathrm{mM}$ potassium phosphate

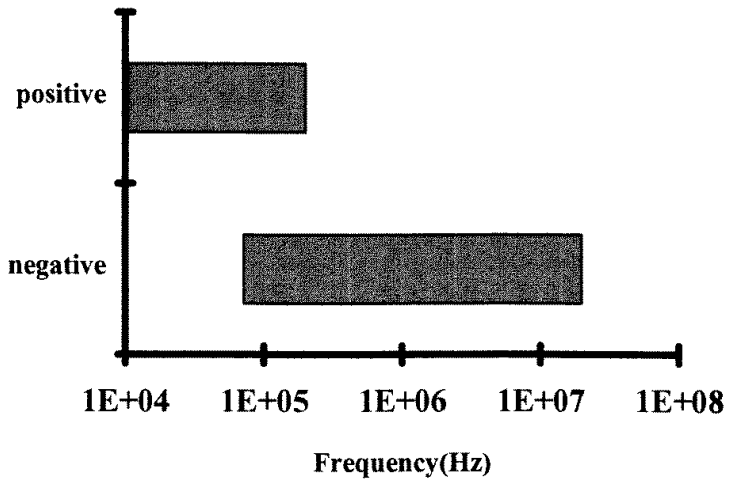

(d) $557 \mathrm{~nm}$ spheres in $10 \mathrm{mM}$ potassium phosphate

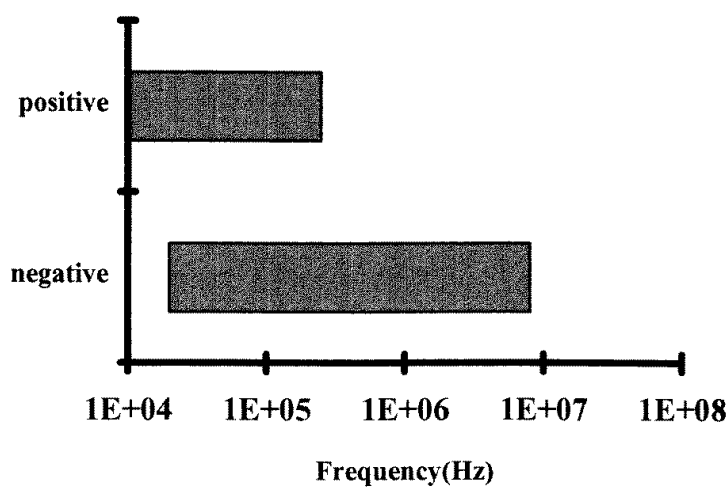

Figure 6. The frequency ranges over which positive and negative DEP are observed for the latex spheres. (a) $282 \mathrm{~nm}$ spheres in $1 \mathrm{mM}$ potassium phosphate: for each particle concentration there is a frequency range around $5 \mathrm{MHz}$ where neither positive nor negative DEP is observed. (b) $282 \mathrm{~nm}$ spheres in $10 \mathrm{mM}$ potassium phosphate: for each particle concentration there is a range around $200 \mathrm{kHz}$ where both positive and negative DEP are observed. (c) $557 \mathrm{~nm}$ spheres in $1 \mathrm{mM}$ potassium phosphate: there is a frequency range around $200 \mathrm{kHz}$ with both positive and negative DEP. (d) $557 \mathrm{~nm}$ spheres in $10 \mathrm{mM}$ potassium phosphate: there is a frequency range around $100 \mathrm{kHz}$ with both positive and negative DEP.

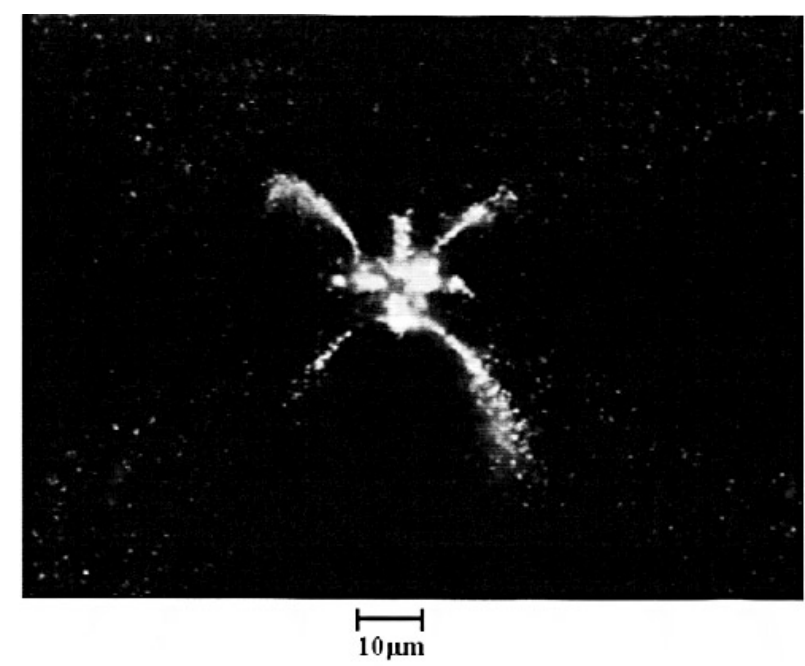

Figure 7. A digitized video image of convection in the polynomial electrodes. The formation of plumes down the symmetry axes of the electrodes is noticeable, occurring particularly at high applied potentials (8-10 V peak-peak).

this regime are the plumes shown in figure 7 , which occur in a low-frequency region where positive DEP is expected but cannot be measured. These effects are both well defined and repeatable and could be useful as an additional means of manipulating particles in solution.

\section{Conclusion}

This paper has demonstrated the potential for dielectrophoretic characterization of sub-micrometre particles in micrometre-scale electrode arrays. The initial experimental results have been shown to match simple theoretical predictions qualitatively. The use of dielectrophoresis for separation, filtration and so on, as has been demonstrated on much larger particles, may therefore be applicable to submicrometre particles. This is of potential interest in areas such as biotechnology for the non-invasive manipulation of viruses and protein molecules.

\section{Acknowledgments}

The authors wish to acknowledge the UK Engineering and Physical Sciences Research Council for the award of a studentship to N G Green and to the UK Biotechnology and Biological Sciences Research Council (grant 17/T05315) 
for supporting this work. We would also like to acknowledge Dr J P H Burt (University of Wales, Bangor) for assistance in preparing figures 3 and 4 .

\section{References}

[1] Pohl H A 1958 J. Appl. Phys. 22 869-71

[2] Pohl H A 1978 Dielectrophoresis (Cambridge: Cambridge University Press)

[3] Pethig R 1991 Automation in Biotechnology ed I Karube (Amsterdam: Elsevier) pp 159-85

[4] Wang X B, Huang Y, Becker F F and Gascoyne P R C 1994 J. Phys. D: Appl. Phys. 27 1571-4

[5] Pethig R 1996 Crit. Rev. Biotechnol. 16 331-48

[6] Markx G H, Huang Y, Zhou X F and Pethig R 1994 Microbiology - UK 140 585-91

[7] Markx G H, Talary M S and Pethig R 1994 J. Biotechnol. 32 29-37

[8] Burt J P H, Pethig R, Gascoyne P R C and Becker F F 1990 Biochim. Biophys. Acta 1034 93-101

[9] Gascoyne P R C, Noshari J, Becker F F and Pethig R 1994 IEEE Trans. Indust. Appl. 30 829-34

[10] Becker F F, Wang X-B, Huang Y, Pethig R, Vykoukal J and Gascoyne P R C 1995 Proc. Natl Acad. Sci. USA 92 $860-4$

[11] Talary M S, Mills K I, Hoy T, Burnett A K and Pethig R 1995 Med. Bio. Eng. Comput. 33 235-7

[12] Mills K I, Stephens M, Talary M, Pethig R and Burnett A K 1995 Blood 86462

[13] Stephens M, Talary M S, Pethig R, Burnett A K and Mills K I 1996 Bone Marrow Transplantation 18 777-82

[14] Burt J P H, Pethig R, Parton A and Dawson D 1995 Inst. Phys. Conf. Ser. 143 381-4

[15] Green N G, Hughes M P, Monaghan W and Morgan H 1997 Microelectron. Eng. 35 421-44

[16] Green N G, Morgan H, Wilkinson C D W and Milner J J 1995 Proc. Meeting of Society for Experimental Biology Animal and Cell Abstracts (St Andrews) p 77

[17] Green N G, Morgan H and Milner J J 1997 Biophys. J. 72 MP448
[18] Green N G and Morgan H 1997 J. Phys. D: Appl. Phys. 30 L41-4

[19] Green N G, Morgan H and Milner J J 1997 J. Biochem. Biophys. Methods at press

[20] Muller T, Gerardino A, Schnelle T, Shirley S G, Bordoni F, De Gasperis G, Leoni R and Fuhr G 1996 J. Phys. D: Appl. Phys. 29 340-9

[21] Schnelle T, Muller T, Fiedler S, Shirley S G, Ludwig K, Hermann A and Fuhr G 1996 Naturwissenschaften 83 $172-6$

[22] Huang Y and Pethig R 1991 Meas. Sci. Technol. 2 1142-6

[23] Wang X-B, Hughes M P, Huang Y, Becker F F and Gascoyne P R C 1995 Biochim. Biophys. Acta 1243 185-94

[24] Russel W B, Saville D A and Schoual W R 1991 Colloidal Dispersions (Cambridge: Cambridge University Press) ch 7

[25] Grahame D C 1947 Chem. Rev. 41 441-501

[26] Hughes M P, Wang X-B, Becker F F, Gascoyne P R C and Pethig R 1994 J. Phys. D: Appl. Phys. 27 1564-70

[27] O'Konski C T 1960 J. Phys. Chem. 64 605-19

[28] Schwarz G 1962 J. Phys. Chem. 66 2636-42

[29] Zhou X-F, Markx G H, Pethig R and Eastwood I M 1995 Biochim. Biophys. Acta 1245 85-93

[30] Pethig R 1979 Dielectric and Electronic Properties of Biological Materials (New York: Wiley)

[31] Schwan H P, Schwarz G, Maczuk J and Pauly H 1962 J. Phys. Chem. 662626

[32] Sasaki S, Ishikawa A and Hanai T 1981 Biophys. Chem. 14 $45-53$

[33] Lyklema J, Duhkin S S and Shilov V N 1983 J. Electroanalytical Chem. Interfacial Electrochem. 143 $1-21$

[34] Springer M M, Korteweg A and Lyklema J 1983 J. Electroanalytical Chem. Interfacial Electrochem. 153 $55-66$

[35] Lyklema J, Springer M M, Shilov V N and Duhkin S S 1986 J. Electroanalytical Chem. Interfacial Electrochem. $19819-26$

[36] Pontiga F and Castellanos A 1992 IEEE Trans. Indust. Appl. 28 520-7 